\title{
Three-Loop Results on the Lattice
}

\author{
B. Allés ${ }^{a *}$, M. Campostrini ${ }^{\mathrm{b}}$, A. Feo ${ }^{\mathrm{b}}$ and H. Panagopoulos ${ }^{\mathrm{b}, \mathrm{c}}$

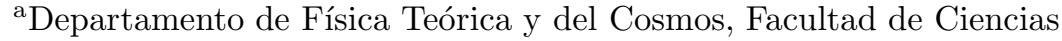 \\ Universidad de Granada, 18071-Granada, Spain \\ ${ }^{\mathrm{b}}$ Dipartimento di Fisica dell'Università and I.N.F.N. \\ Piazza Torricelli 2, 56126-Pisa, Italy \\ ${ }^{\mathrm{c}}$ Department of Physics, University of Cyprus, Nicosia, Cyprus
}

We present some new three-loop results in lattice gauge theories, for the Free Energy and for the Topological Susceptibility. These results are an outcome of a scheme which we are developing (using a symbolic manipulation language), for the analytic computation of renormalization functions on the lattice.

\section{INTRODUCTION}

The computation of vacuum expectation values of composite operators is one of the most important tasks in lattice gauge theories. Examples of such expectation values in QCD are the Condensates which are essential tools for the SVZ sum rules [1] or the Topological Susceptibility which is necessary to solve the so-called $U_{A}(1)$ problem and to understand the $\eta^{\prime}$ mass [2 2 . In this talk we present some results related to the computation on the lattice of the Gluon Condensate

$G_{2} \equiv\left\langle 0\left|: \frac{g^{2}}{4 \pi^{2}} G_{\mu \nu}^{b} G_{\mu \nu}^{b}:\right| 0\right\rangle$,

and the Topological Susceptibility $\chi$

$\chi \equiv \int \mathrm{d}^{4} x\langle 0|\mathrm{~T}(Q(x) Q(0))| 0\rangle$,

where $Q(x)$ is the Topological Charge density

$Q(x) \equiv \frac{g^{2}}{64 \pi^{2}} \epsilon^{\mu \nu \rho \sigma} G_{\mu \nu}^{b} G_{\rho \sigma}^{b}$.

In these equations, $g$ is the coupling constant of the QCD lagrangian and $G_{\mu \nu}^{b}$ the strength tensor for the gluon fields.

To evaluate one of these quantities on the lattice, e. g. $\langle 0|\mathcal{A}| 0\rangle$, one defines first a lattice version $\mathcal{A}^{L}$ of $\mathcal{A}$ in such a way that $\mathcal{A}^{L} \stackrel{a \rightarrow 0}{\longrightarrow} a^{d} \mathcal{A}+$

\footnotetext{
*Presented the talk.
}

$\mathcal{O}\left(a^{d+1}\right)$ where $d$ is the mass dimension of the operator $\mathcal{A}$ and $a$ is the lattice spacing. For the Gluon Condensate and Topological Susceptibility respectively, our choices for these lattice versions are

$$
\begin{aligned}
G_{2}^{L} & \equiv\left\langle 0\left|1-\Pi_{\mu \nu}\right| 0\right\rangle, \\
\chi^{L} & \equiv\left\langle 0\left|\sum_{x} Q^{L}(x) Q^{L}(0)\right| 0\right\rangle,
\end{aligned}
$$

where $Q^{L}(x)$ is a lattice version of the Topological Charge density [5]

$$
\begin{gathered}
Q^{L}(x)=-\frac{1}{2^{4} 32 \pi^{2}} \sum_{\mu, \nu, \rho, \sigma= \pm 1}^{ \pm 4} \epsilon_{\mu \nu \rho \sigma} \times \\
\operatorname{Tr}\left[\Pi_{\mu \nu}(x) \Pi_{\rho \sigma}(x)\right] .
\end{gathered}
$$

In these equations, $\Pi_{\mu \nu}$ is the usual plaquette in the $\mu-\nu$ plane.

Once the lattice version of the operator is defined, one can perform a Monte Carlo simulation. The Monte Carlo data will give the physical continuum value of $\langle 0|\mathcal{A}| 0\rangle$ modified by some $a$-dependent renormalizations. These expressions for the Gluon Condensate and Topological Susceptibility can be written in the following way [6] 8]

$$
\begin{aligned}
& G_{2}^{L}=\frac{\pi^{2}}{12 N} Z_{G} a^{4} G_{2}+\sum_{n \geq 1} \frac{c_{n}}{\beta^{n}}, \\
& \chi^{L}=Z_{Q}^{2} a^{4} \chi+a^{4} G_{2} \sum_{n \geq 2} \frac{b_{n}}{\beta^{n}}+\sum_{n \geq 3} \frac{d_{n}}{\beta^{n}},
\end{aligned}
$$


where $N$ is the number of colors and as usual $\beta=$ $2 N / g^{2}$. The lattice spacing $a$ and $\beta$ are related by the renormalization group equation

$a \Lambda_{L}=\left(\frac{2 N r_{0}}{\beta}\right)^{-r_{1} / 2 r_{0}^{2}} \exp \left(-\frac{\beta}{4 N r_{0}}\right)$,

where $\Lambda_{L}$ is the renormalization group invariant mass parameter of QCD and $r_{0}$ and $r_{1}$ the first two coefficients of the $\beta$ function, $\beta(g)=-r_{0} g^{3}-$ $r_{1} g^{5}-\ldots$

The last terms in these expressions (those proportional to $c_{n}$ and $d_{n}$ ) are the perturbative tails and represent mixings with the unity operator. The second term in Eq.(7) is a mixing with the Gluon Condensate. Finally, $Z_{G}$ and $Z_{Q}$ are multiplicative finite renormalizations which relate the lattice and the continuum definitions of the respective operators. All of these coefficients can be computed in perturbation theory and their knowledge is essential to extract the physical values $G_{2}$ and $\chi$ from the Monte Carlo data and Eq.(6-7). The values of these coefficients also depend on the lattice versions used for the operators and the lattice action chosen. Throughout this work we have used the lattice versions shown in Eq.(4) and the Wilson action.

\section{RENORMALIZATION CONSTANTS}

The first coefficients in the renormalization terms of Eq.(6-7) can be calculated with rather small effort because they involve very few Feynman diagrams. The result for $S U(3)$ is 60 [10]

$$
\begin{aligned}
& Z_{G}=1+\mathcal{O}\left(1 / \beta^{2}\right), \\
& Z_{Q}=1-5.45 / \beta+\mathcal{O}\left(1 / \beta^{2}\right) \\
& b_{2}=6.32 \times 10^{-3} \quad d_{3}=3.58 \times 10^{-3} \\
& c_{1}=2.0 \quad c_{2}=1.22 .
\end{aligned}
$$

However, the next coefficients involve many more diagrams. For instance, $c_{1}$ and $c_{2}$ are calculated with three diagrams, but the next order, $c_{3}$, needs the computation of 30 three-loop Feynman diagrams! To evaluate these Feynman diagrams we have developed an algebraic computer program. Major tasks in this algorithm are:

i) Computing n-point vertices.

ii) Producing a list of relevant diagrams, with the corresponding weights. iii) Performing the contractions for each diagram, using up all existing symmetries to produce a compact result.

$i v$ ) Extracting the analytic dependence of each diagram on its external momenta $p$, in the limit $a p \rightarrow 0$.

$v$ ) Producing the optimized code for the numerical calculation of the loop integrals.

Regarding the first step, some difficulties inherent to the lattice are: The existence of vertices with an arbitrary number of gluons, a plethora of "tensor" structures (due to lack of rotational invariance) and a great proliferation in the size of vertices (a 6-point vertex in its most compact form may typically require some dozens of output pages). This in turn necessitates, in the third step, simplifying all intermediate expressions as much as possible, by devising algorithms which use up the symmetries of the diagram under exchange of external legs, under allowed redefinitions of momenta and under permutation of the (numerous) dummy indices.

The fourth step is necessary for computing multiplicative renormalizations. The evaluation of these form factors is in progress.

Now the final expression for the diagram can be integrated. With the code produced by the algorithm, we can calculate the numerical value of the diagram for rather small lattices and then extrapolate for larger lattices. This extrapolation is performed by assuming the following dependence of the diagram on the lattice size $L$

diagram $=A+\frac{B}{L^{n}}$.

The explicit values for $A, B$ as well as that of $n$ (which may vary for different diagrams) are determined by the extrapolation. The result is then confronted with the results for an infinite lattice, which we also compute. We will explain this computation for an infinite lattice with an example. Let us consider the following integral

$I=\int_{-\pi}^{+\pi} \frac{1}{\hat{p}^{2}} \frac{1}{\hat{q}^{2}} \frac{1}{p \hat{+} q^{2}} \frac{\mathrm{d}^{4} p}{(2 \pi)^{4}} \frac{\mathrm{d}^{4} q}{(2 \pi)^{4}}$

which is the value of a two-loop diagram for an infinite lattice. In this expression, $\hat{p}^{2}=2 \sum_{\mu}(1-$ 
Table 1

Exact and fitted values for $c_{3}$ and $d_{4}$

\begin{tabular}{ccccc}
\hline & \multicolumn{2}{c}{$S U(2)$} & \multicolumn{2}{c}{$S U(3)$} \\
\cline { 2 - 5 } & Exact & Fitted & Exact & Fitted \\
\hline$c_{3}$ & 0.16 & 0.18 & 3.12 & 3.0 \\
$d_{4}$ & $7.0 \times 10^{-5}$ & $1.8(6) \times 10^{-4}$ & $8.4 \times 10^{-4}$ & $1.6(1.0) \times 10^{-3}$ \\
\hline
\end{tabular}

$\left.\cos p_{\mu}\right)$. Using the Schwinger representation for the propagators,

$\frac{1}{\hat{p}^{2}}=\int_{0}^{\infty} \mathrm{e}^{-\alpha \hat{p}^{2}} \mathrm{~d} \alpha$,

we get

$I=\int_{0}^{\infty} \mathrm{d} \alpha_{1} \int_{0}^{\infty} \mathrm{d} \alpha_{2} \int_{0}^{\infty} \mathrm{d} \alpha_{3} \Phi^{4}\left(\alpha_{1}, \alpha_{2}, \alpha_{3}\right)$

where $\Phi$ is

$$
\begin{aligned}
& \Phi\left(\alpha_{1}, \alpha_{2}, \alpha_{3}\right)= \\
& \mathrm{e}^{-2\left(\alpha_{1}+\alpha_{2}+\alpha_{3}\right)} \int_{-\pi}^{+\pi} \frac{\mathrm{d} p}{2 \pi} \int_{-\pi}^{+\pi} \frac{\mathrm{d} q}{2 \pi} \times \\
& \exp \left(2 \alpha_{1} \cos p+2 \alpha_{2} \cos q+2 \alpha_{3} \cos (p+q)\right)= \\
& \mathrm{e}^{-2\left(\alpha_{1}+\alpha_{2}+\alpha_{3}\right)} \int_{-\pi}^{+\pi} \frac{\mathrm{d} p}{2 \pi} \times \\
& \mathrm{e}^{2 \alpha_{2} \cos p} \quad \mathrm{I}_{0}\left(2 \sqrt{\alpha_{1}^{2}+\alpha_{3}^{2}+2 \alpha_{1} \alpha_{3} \cos p}\right) .
\end{aligned}
$$

In Eq.(14) $I_{0}$ is the modified Bessel function. Therefore we have reduced the number of integration variables from 8 in Eq.(11) to 4 in Eq.(13). For three-loop integrals with 12 integration variables, this procedure reduces this number to 6 integrations (for a few cases it only reduces to 8 integrations). Now, a numerical integration is feasible.

The extrapolation done with Eq.(10) is enough to obtain the correct values of the coefficients with three digits. Further details of the algorithm will be published elsewhere.

\section{RESULTS}

Summing up the individual contributions of all diagrams we obtain for the gauge group $S U(N)$ and an infinite lattice [11]

$d_{4}=N^{4}\left(N^{2}-1\right)\left(1.73 N^{2}-10.83+\frac{73.83}{N^{2}}\right) 10^{-7},(15)$
$c_{3}=N^{2}\left(N^{2}-1\right)\left(7.28 N^{2}-27.35+\frac{46.15}{N^{2}}\right) 10^{-3} \cdot(16)$

The exact values for the coefficients obtained with our scheme can be compared with the values extracted by a best fit of all Monte Carlo data with Eqs.(6-9). Both values are shown in table 1 for the coefficients $d_{4}$ and $c_{3}$ and for an infinite lattice 6 6.12 . 1 .

The agreement between fitted and exact results is manifest for $c_{3}$. The data for the Topological Susceptibility had a rather low statistics, therefore and within the errors the numbers shown in table 1 for $d_{4}$ are in acceptable agreement.

Finally, we can add the exact values of $c_{3}$ and $d_{4}$ to those of Eq.(9) and perform again the best fits. The values extracted for $G_{2}$ and $\chi$ are in complete agreement with those reported in refs. 68.12].

\section{ACKNOWLEDGMENTS}

We wish to thank Adriano Di Giacomo for useful conversations and the spanish-italian "Acción Integrada/Azione Integrata" number $A 17$ for financial support. B. A. also acknowledges a spanish CICYT contract.

\section{REFERENCES}

1. M. A. Shifman, A. I. Vainshtein and V. I. Zakharov, Nucl. Phys. B147 (1979) 385, 448, 519.

2. G. t'Hooft, Phys. Rev. Lett. 37 (1976) 8; Phys. Rev. D14 (1976) 3432.

3. E. Witten, Nucl. Phys. B156 (1979) 269.

4. G. Veneziano, Nucl. Phys. B159 (1979) 213.

5. P. Di Vecchia, K. Fabricius, G. C. Rossi and

\footnotetext{
${ }^{1}$ Actually, for the Topological Susceptibility we calculate the unrenormalized value $\chi_{u}$ because the physical one is better obtained by using the cooled data of ref. [12].
} 
G. Veneziano, Nucl. Phys. B192 (1981) 392; Phys. Lett. B108 (1982) 323.

6. M. Campostrini, G. Curci, A. Di Giacomo and G. Paffuti, Z. Phys. C32 (1986) 377.

7. M. Campostrini, A. Di Giacomo and Y. Gündüç, Phys. Lett. B225 (1989) 393.

8. M. Campostrini, A. Di Giacomo, H. Panagopoulos and E. Vicari, Nucl. Phys. B329 (1990) 683.

9. B. Allés and M. Giannetti, Phys. Rev. D44 (1991) 513.

10. M. Campostrini, A. Di Giacomo and H. Panagopoulos, Phys. Lett. B212 (1988) 206.

11. B. Allés, M. Campostrini, A. Feo and H. Panagopoulos, Lattice Perturbation Theory Done Symbollically: A Three-Loop Result for the Topological Susceptibility, Pisa preprint IFUP-TH-31/92; The Three-Loop Lattice Free Energy, Pisa preprint IFUP-TH$32 / 92$.

12. M. Campostrini, A. Di Giacomo, Y. Gündüç, M. P. Lombardo, H. Panagopoulos and R. Tripiccione, Phys. Lett. B252 (1990) 436. 